\title{
Structural Abnormalities in Patients with Insular/Peri-insular Epilepsy: Spectrum, Frequency, and Pharmacoresistance
}

\author{
M.-C. Chevrier, C. Bard, F. Guilbert, and D.K. Nguyen
}

\begin{abstract}
SUMMARY: Between 2002 and 2010, a total of 48 patients were seen at our epilepsy clinic with insular/peri-insular cortex epilepsy. Review of their MR imaging scans revealed a neoplastic lesion in $27 \%$ of patients, a malformation of cortical development in $21 \%$, a vascular malformation in 19\%, and atrophy/gliosis from an acquired insult in 17\%. MR imaging results were normal in 4 patients. Other miscellaneous findings included a case of Rasmussen encephalitis, a nonspecific insular millimetric T2 signal abnormality, a neuroepithelial cyst, and hippocampal sclerosis without MR imaging evidence of dual insular pathologic features (despite depth electrode-proven insular seizures). Refractoriness to antiepileptic drug treatment was present in $56 \%$ of patients: $100 \%$ for patients with malformations of cortical development (1.0; $95 \% \mathrm{Cl}, 0.72-1.0), 50.0 \%(0.5 ; 95 \% \mathrm{Cl}, 0.21-0.78)$ in the presence of atrophy/gliosis from acquired insults, 39\% $(0.39 ; 95 \% \mathrm{Cl}$, $0.14-0.68)$ for neoplastic lesions, and $22.2 \%(0.22 ; 95 \% \mathrm{Cl}, 0.06-0.55)$ for vascular malformations.
\end{abstract}

ABBREVIATIONS: IPICE = insular/peri-insular cortex epilepsy; MCD = malformation of cortical development

$\mathbf{S}$ ructural abnormalities underlying temporal and frontal lobe epilepsies have been well described. ${ }^{1,2}$ Little is known about the type and frequency of structural brain abnormalities that give rise to insular/peri-insular cortex epilepsy (IPICE). ${ }^{3}$ The insula is a highly developed structure, totally encased within the brain in the depths of the Sylvian fissure and covered by the frontal, parietal, and temporal opercula. ${ }^{4}$ Insular seizures are typically associated with a sensation of laryngeal constriction and paresthesias affecting perioral or large cutaneous territories with preserved consciousness, followed by dysarthric speech and/or elementary auditory hallucinations or motor signs. ${ }^{5}$ Later observations have also showed that insular seizures could feature hypermotor symptoms resembling frontal lobe seizures, ${ }^{6}$ early visceral signs or dysphasia with subsequently altered consciousness suggesting temporal lobe seizures, and early somatosensory symptoms in the absence of laryngeal constriction mimicking parietal lobe sei-

Received August 8, 2012; accepted after revision February 5, 2013.

From the Radiology Department (M.-C.C., C.B., F.G.) and Neurology Service (D.K.N.), Centre hospitalier de l'Université de Montréal (CHUM) - Hôpital Notre-Dame and Université de Montréal, Montreal, Quebec, Canada.

Paper previously presented as a scientific poster at: Annual Meeting of the Radiological Society of North America, November 28-December 3, 2010; Chicago, Illinois.

Please address correspondence to Dang K. Nguyen, MD, FRCPC, Service de neurologie, CHUM - Hôpital Notre-Dame, 1560 rue Sherbrooke est, Montréal, Québec H2L 4M1, Canada; e-mail: d.nguyen@umontreal.ca

三 Indicates article with supplemental on-line table.

http://dx.doi.org/10.3174/ajnr.A3636 zures. $^{7}$ The purpose of our study was to ascertain 1) the overall type and distribution of structural abnormalities, identified by MR imaging, in association with IPICE; and 2) their refractoriness to antiepileptic drug therapy.

\section{CASE SERIES}

\section{Materials and Methods}

Charts from all patients seen at the epilepsy clinic by one of the authors (D.K.N.) from August 2002-May 2010 were reviewed retrospectively to find patients with IPICE. Patients were classified as having 1) definite IPICE when insular seizures were confirmed by intracerebral recordings; 2) probable IPICE in partial epilepsy with early ictal somatosensory symptoms and/or a sensation of laryngeal constriction associated with insular/peri-insular lesions; and 3) possible IPICE in partial epilepsy with early viscerosensory symptoms, speech arrest, dysphasia, or hypermotor ictal manifestations coupled with insular/peri-insular lesions on MR imaging or clear insular activation on ictal SPECT. Pharmacoresistance was defined as failure of the epilepsy to be controlled with 3 appropriate antiepileptic drug trials.

Imaging protocols varied because this study was retrospective, and some imaging was performed at referring centers. Most were standard MR imaging protocols, tumor protocols, or epilepsy protocols. MR imaging scans of all patients with IPICE were reviewed retrospectively by a neuroradiologist with 15 years of experience and a senior resident in radiology. The following features were noted: type of structural abnormality, anatomic location, and extent. 
For statistical analysis, patients were partitioned within 6 radiopathologic categories: 1 (neoplastic), 2 (malformations of cortical development [MCDs], 3 (vascular malformations), 4 (atrophy/gliosis from acquired insults), 5 (other), and 6 (normal). A $2 \times 6$ contingency table was constructed partitioning patients between pharmacoresistant and responsive to medication. A Fisher exact test was performed on this $2 \times 6$ contingency table assuming that categories are mutually exclusive, followed by a Fisher exact test for each category when appropriate. A Bonferroni correction was used to control for multiplicity ( $\alpha=0.05 / 6$ or $\alpha=0.008$ ). For each category presenting a significant association with pharmacoresistance, the risk was reported with a 95\% CI. Calculations were produced on StatsDirect statistical software (version 2.7.9, http://www.statsdirect.com).

\section{RESULTS}

A total of $59(6.4 \%)$ of 920 patients with epilepsy had IPICE. Eleven patients were excluded because MR imaging scans of 5

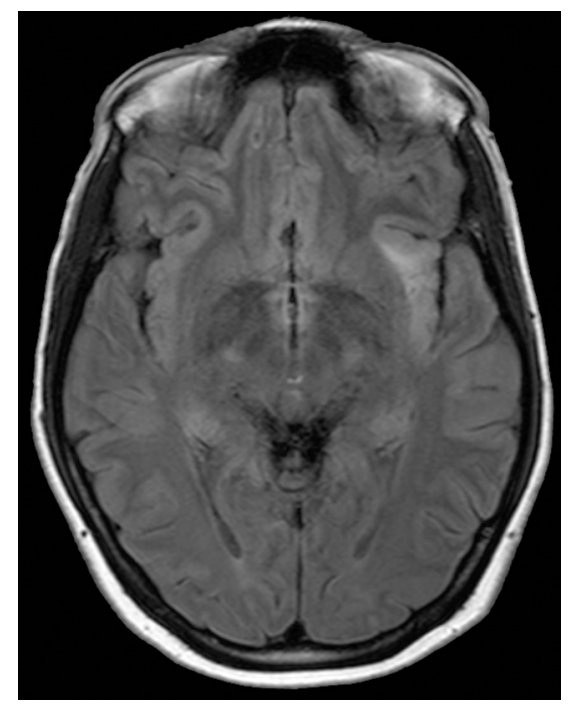

FIG 1. Axial FLAIR MR imaging scan of a 24-year-old woman with low-grade glioma in whom left IPICE (probable) developed. A hyperintense FLAIR lesion can be seen infiltrating the left insula.
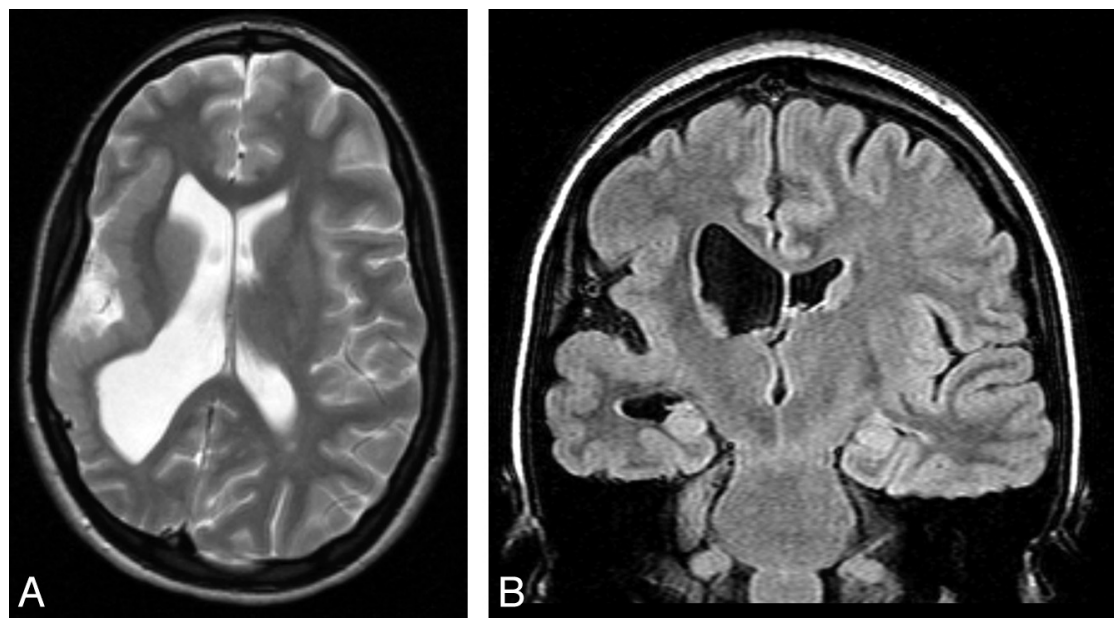

FIG 2. Coronal FLAIR and axial T2 MR imaging scans of a 19-year-old woman with epilepsy with polymicrogyria in the right insula and postcentral gyrus. patients were destroyed, no MR imaging scans were taken of 2 patients because of contraindications (eg, pacemaker), and MR imaging was not available before surgical treatment in 4 patients. Hence, we retrospectively reviewed the MR imaging results of 48 patients with IPICE. Of these patients, 7 had definite IPICE, 24 had probable IPICE, and 17 had possible IPICE. The group included 21 men and 27 women with a median age of 39 years (age range, $2-73$ years).

The On-line Table lists the pathologic patterns detected on MR imaging in the 48 patients and refractoriness to antiepileptic drug treatment. It disclosed neoplastic lesions in 13 patients (27\%). Eight patients presented pathologic features compatible with low-grade glioma and 5 patients, with high-grade glioma. Eight patients had astrocytomas (Fig 1), 4 of which were lowgrade, and 4 were high-grade. Three patients had low-grade oligoastrocytoma. MR imaging results in these patients demonstrated infiltrative masses centered over the insula with extension to the frontal and temporal lobes. One patient, who had a previously resected, low-grade oligodendroglioma, went on to have IPICE many years later in the context of insular tumor recurrence. Another patient had glioblastoma multiforme involving the insula and the temporal operculum.

MCDs were detected in 10 patients (20\%). Five patients had cortical dysplasia involving the insula; 4 had polymicrogyria implicating the insula with perisylvian extension (Fig 2), including 1 patient with bilateral perisylvian polymicrogyria; and another patient had tuberous sclerosis presenting multiple cortical tubers, 1 being located in the operculoinsular region (Fig 3). Vascular malformations were found in 9 patients (19\%). Four patients had an arteriovenous malformation (Fig 4) with a nidus in the insula or inducing a FLAIR signal abnormality in the insula. Three patients had a Sylvian bifurcation aneurysm with mass effect on the insular cortex, and 2 others had a cavernoma centered on the insula (Fig 5).

Atrophy/gliosis from acquired insults was observed in 8 patients (17\%). Two patients had encephalomalacia and atrophy after trauma (Fig 6): one involving the temporal operculum and insula after surgical evacuation of a hematoma, and the other involving the insula and frontal and temporal opercula. One patient had signs of an old ischemic infarct exclusively in the insular cortex. Another patient had cystic encephalomalacia after herpes encephalitis affecting the insula and temporal lobes. Yet another patient had encephalomalacia and atrophy after a congenital insult, 2 patients had encephalomalacia and atrophy of indeterminate cause involving the insula and the frontal or temporal opercula, and 1 other patient had pial enhancement over the insula and frontal operculum of unknown cause.

Serial MR imaging in a patient with Rasmussen encephalitis revealed an initial insular FLAIR signal abnormality followed by unilateral fronto-temporoinsular atrophy and gliosis. A millimetric, innocuous-looking T2 signal abnormality over the posterior insula was 

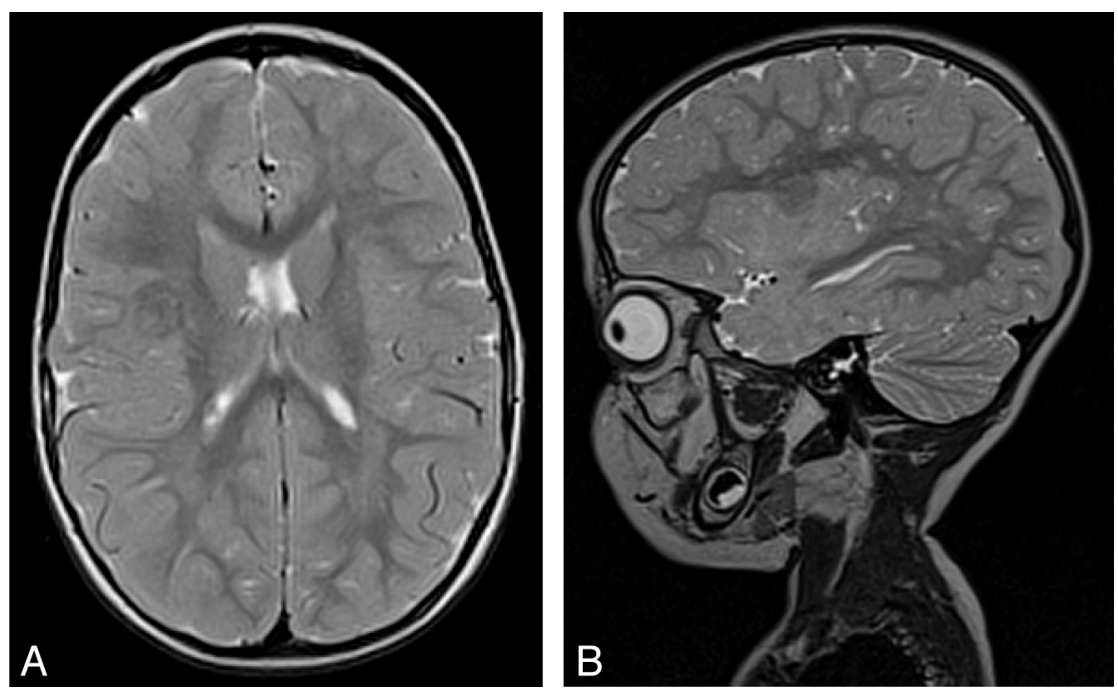

FIG 3. Axial and sagittal T2 MR imaging scan of a 2-year-old girl with tuberous sclerosis in whom right insular epilepsy (definite) developed. Cortical tuber involving the short posterior and long anterior gyri of the insula. Note the white matter abnormalities of tuberous sclerosis.
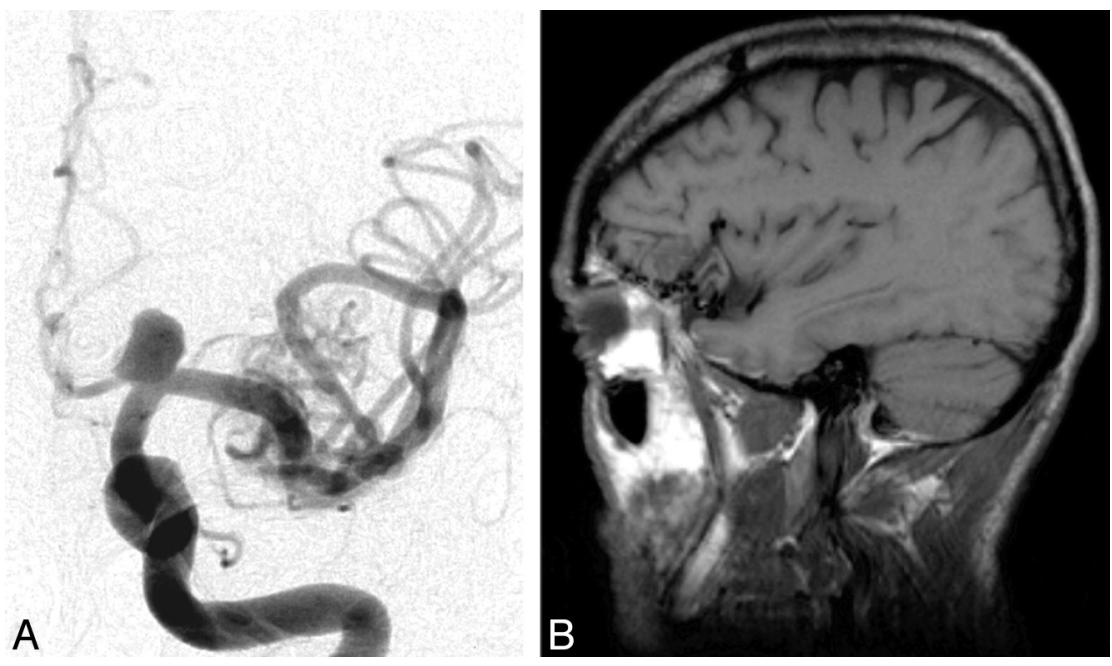

FIG 4. Angiography of the left carotid artery with sagittal TI MR imaging scan of a 54-year-old man with left fronto-insular epilepsy (probable). Angiography shows an arteriovenous malformation fed by the left middle cerebral artery and a left supraclinoid internal carotid artery aneurysm. MR imaging scan reveals the arteriovenous malformation centered on the left frontal operculum with mass effect on the anterior short gyrus of the insula.

found in 1 patient. A neuroepithelial cyst vs a signal abnormality in the insula was seen in another patient. One patient had no insular lesions (despite depth electrode-proven insular seizures) but rather hippocampal sclerosis (and concomitant hippocampal seizures). MR imaging results were normal in 4 patients: 2 of whom had definite IPICE, and 2 of whom had possible IPICE.

Among the 43 patients with lesions in the insula, damage was confined to the insular cortex with or without involvement of the adjacent operculum in 20 patients. More than 1 operculum was affected in the remaining 23 patients.

Refractoriness to antiepileptic drug treatment was $100 \%$ for the 10 patients with MCD (1.0; 95\% CI, 0.72-1.0), 50.0\% (0.5; 95\% CI, 0.21-0.78) in the presence of atrophy/gliosis from acquired insults, 39\% $(0.39 ; 95 \%$ CI, $0.14-0.68)$ for neoplastic lesions, and $22.2 \%(0.22$; $95 \% \mathrm{CI}, 0.06-0.55)$ for vascular mal- formations. An estimated $75 \%$ of patients with normal MR imaging results (0.75; 95\% CI, 0.19-0.99) had refractory epilepsy (Table). The patient with Rasmussen encephalitis had refractory epilepsia partialis continua with occasionally complex partial seizures.

\section{DISCUSSION}

Some epileptogenic lesions are more commonly found in certain regions of the brain. For example, typical epileptogenic lesions diagnosed in patients with temporal lobe epilepsy are hippocampal atrophy/sclerosis, dysembryoplastic neuroepithelial tumors, gangliogliomas, postherpetic encephalitic gliosis, and posttraumatic encephalomalacia. ${ }^{2,8,9}$ Until now, little was known about the epileptogenic lesions associated with IPICE. In our study, 13 patients (27\%) with IPICE manifested tumors, 8 of whom had low-grade gliomas. Duffau et $\mathrm{al}^{10}$ had previously reported that the insular cortex itself may induce chronic seizures when injured by tumors. In 2010 , Lee et $\mathrm{al}^{11}$ observed that $34 \%$ of patients with high-grade tumors and $62 \%$ of those with low-grade tumors had epilepsy. Among patients with lowgrade gliomas, those with tumors located in the temporal lobe as well as in the insular region were more likely to present with seizures. MCDs constitute $4 \%-25 \%$ of all epileptogenic lesions in adults and $10 \%-50 \%$ in pediatric populations. ${ }^{12-14}$ Our study determined that 10 patients $(20.8 \%)$ with IPICE had MCDs, 4 (8.3\%) of whom had perisylvian polymicrogyria. This preferential localization in the perisylvian area for polymicrogyria has been well demonstrated by Leventer et al, ${ }^{15}$ who found this pattern in 200 (61\%) of 328 patients. Cortical tubers, which histologically resemble cortical dysplasias, can also induce seizures in this zone. ${ }^{16}$

Nine patients $(18.8 \%)$ with IPICE had vascular lesions. By its very location, the insula is susceptible to vascular lesions. ${ }^{17}$ It is closely related to the Sylvian artery, from which it receives its blood supply via numerous perforating arteries from the M2 and M3 segments. As reported by Malak et al, ${ }^{18}$ unruptured Sylvian bifurcation aneurysms can present with a combination of viscerosensitive, motor, language, autonomic, and somatosensory symptoms related to the proximity of the insula. Arteriovenous malformations and cavernomas can also cause IPICE by direct compression or hemorrhage. Any previous intracranial insult can result in epilepsy. In our series, 8 patients (17\%) with IPICE had 

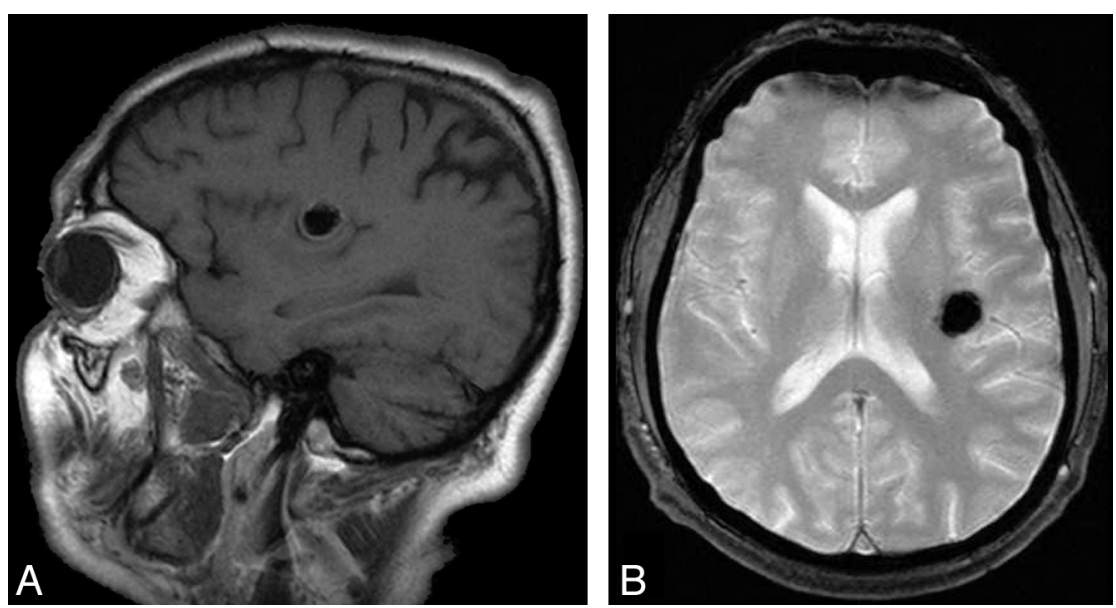

FIG 5. Sagittal TT and gradient-echo MR imaging of a 64-year-old man with a left insular cavernoma in whom left insular epilepsy (definite) developed. Round, hypointense T1, T2, and T2* lesion involves the anterior and posterior long gyri of the insula.
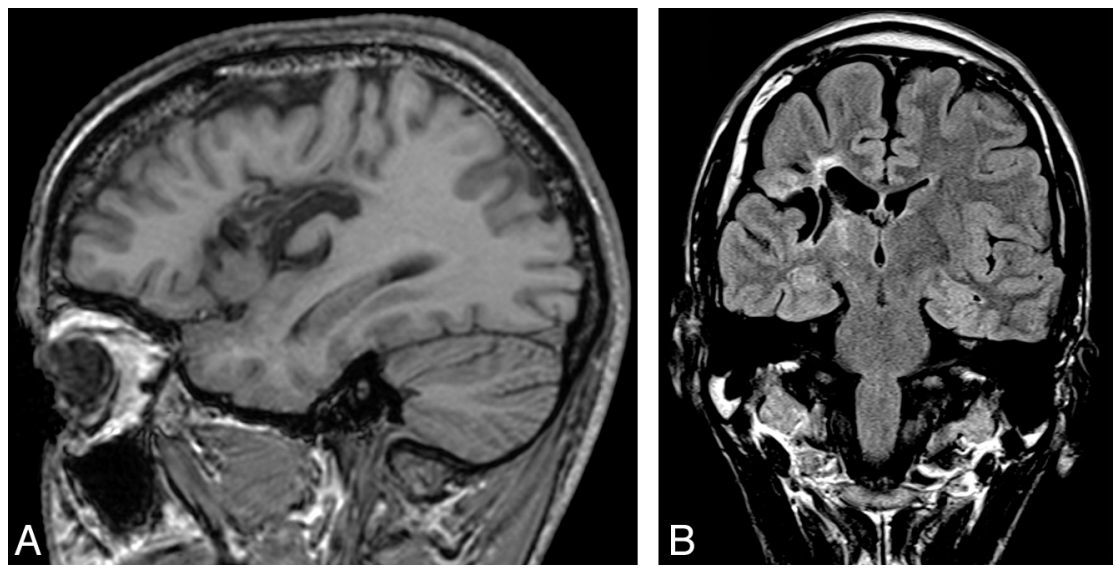

FIG 6. Sagittal T1 and coronal FLAIR MR imaging of a 53-year-old man with a history of trauma in whom right fronto-temporo-insular epilepsy (definite) developed. Encephalomalacia and atrophy involve the insula, frontal operculum, and superior temporal gyrus.

Distribution of patients according to radiopathologic categories and refractoriness to medication

\begin{tabular}{|c|c|c|c|}
\hline \multirow[b]{2}{*}{ Pathologic Features } & \multicolumn{2}{|c|}{ Medication } & \multirow[b]{2}{*}{$P$ Value } \\
\hline & Response (n) & Refractoriness (n) & \\
\hline Neoplastic lesion & 8 & 5 & .19 \\
\hline Malformations of cortical development & 0 & 10 & $.001^{\mathrm{a}}$ \\
\hline Vascular lesions & 7 & 2 & .03 \\
\hline Atrophy/gliosis from acquired insults & 4 & 4 & .71 \\
\hline Other & 1 & 3 & .49 \\
\hline Normal & 1 & 3 & .62 \\
\hline Total & 21 & 27 & $.004^{\mathrm{b}}$ \\
\hline
\end{tabular}

${ }^{a}$ Statistical significance threshold after Bonferroni method, $P=.008$

${ }^{\mathrm{b}} P$ value obtained by Fisher exact test on the overall $2 \times 6$ contingency table (statistical significance threshold $P=.05$ ). with Rasmussen encephalitis starts preferentially in the insula and in the perisylvian area. ${ }^{21-23}$

Refractoriness to antiepileptic drug treatment was $100 \%$ for MCDs in the insular/peri-insular region, which is not necessarily surprising, as such entities located in other areas are generally linked with drug-resistant epilepsies as well. ${ }^{3}$ The refractoriness rate was $38.5 \%$ (5/13) for patients with tumors in our series, most of which were low-grade gliomas $(4 / 5 ; 80 \%)$. These findings are consistent with the observations of Duffau et al, ${ }^{10}$ who recorded a refractoriness rate of $58 \%$ with insular low-grade gliomas and suggested that the latter are more prone to eliciting drug-resistant epilepsy than tumors invading other brain structures. ${ }^{10,24}$ Although half (2/4) of patients with IPICE related to atrophy/gliosis had drug-resistant seizures, their low number did not allow further interpretation. Although some studies have reported a high rate of drug-resistant seizures in posttraumatic $^{25,26}$ or postencephalitic epilepsies, ${ }^{27}$ epilepsies after strokes usually respond to medical treatment. ${ }^{28}$ Of interest, 1 patient with a millimetric T2 signal abnormality in the posterior insula (initially dismissed as nonspecific), 1 patient with hippocampal sclerosis but no insular lesion, and 3 of 4 patients with normal MR imaging results had refractory insular seizures confirmed by invasive recordings. This finding raises the possibility that a certain proportion of patients with persisting seizures, despite temporal, frontal, or parietal lobe epilepsy surgeries, might have unrecognized IPICE. $^{29,30}$

The limitations of our study are inherent to any retrospective design. Because MR imaging scans were not standardized (magnet strength, acquisition protocols), anatomic delineation of insular involvement was constrained in atrophy or encephalomalacia from acquired insults. Trauma and herpes encephalitis classically injure the temporal lobes, but damage may extend to the insula. Although strokes are the most common source of epilepsy in elderly people, they were not found here to be a cause of IPICE, likely because of the rare occurrence of pure insular ischemic strokes. ${ }^{19}$ One patient with IPICE had Rasmussen encephalitis, a chronic inflammatory disease of unknown origin affecting 1 hemisphere. ${ }^{20}$ Some imaging studies have previously suggested that the progressive hemiatrophy associated some cases. Coronal and sagittal $\mathrm{T} 2$-weighted sequences with thin sections on the insula would certainly have increased the precision. Although the lesions were centered on the insular/peri-insular area, some extended slightly beyond, and we cannot exclude that seizures originated from adjacent lobes with rapid propagation to the insular/peri-insular area. Finally, selecting patients from a specialized epilepsy clinic in a tertiary academic center may have biased the study into finding a higher rate of lesions associated with intractable epilepsy. 


\section{CONCLUSIONS}

A spectrum of structural abnormalities may evoke IPICE. In order of frequency, we noted tumors, MCDs, vascular lesions, and atrophy/gliosis from acquired insults. IPICE related to MCDs, atrophy/gliosis from acquired insults, and tumors is frequently refractory to antiepileptic drugs.

\section{REFERENCES}

1. Chugani HT. Neuroimaging in Epilepsy. New York: Oxford University Press; 2011:3-37

2. Cakirer S, Başak M, Mutlu A, et al. MR imaging in epilepsy that is refractory to medical therapy. Eur Radiol 2002;12:549-58

3. Surbeck W, Bouthillier A, Nguyen DK. Refractory insular cortex epilepsy: clinical features, investigation and treatment. Future Neurol 2010;5:491-99

4. Naidich TP, Kang E, Fatterpekar GM, et al. The insula: anatomic study and MR imaging display at 1.5T. AJNR Am J Neuroradiol 2004;25:222-32

5. Isnard J, Guenot M, Sindou M, et al. Clinical manifestations of insular lobe seizures: a stereo-electroencephalographic study. Epilepsia 2004;45:1079-90

6. Ryvlin P, Minotti L, Demarquay G, et al. Nocturnal hypermotor seizures, suggesting frontal lobe epilepsy, can originate in the insula. Epilepsia 2006;47:755-65

7. Nguyen DK, Nguyen DB, Malak R, et al. Revisiting the role of the insula in refractory partial epilepsy. Epilepsia 2009;50:510-20

8. Lefkopoulos A, Haritanti A, Papadopoulou E, et al. Magnetic resonance imaging in 120 patients with intractable partial seizures: a preoperative assessment. Neuroradiology 2005;47:352-61

9. Zentner J, Hufnagel A, Wolf HK, et al. Surgical treatment of temporal lobe epilepsy: clinical, radiological, and histopathological findings in 178 patients. J Neurol Neurosurg Psychiatry 1995;58:666-73

10. Duffau H, Capelle L, Lopes M, et al. Medically intractable epilepsy from insular low-grade gliomas: improvement after an extended lesionectomy. Acta Neurochir (Wien) 2002;144:563-72; discussion $572-73$

11. Lee JW, Wen PY, Hurwitz S, et al. Morphological characteristics of brain tumors causing seizures. Arch Neurol 2010;67:336-42

12. Brodtkorb E, Nilsen G, Smevik O, et al. Epilepsy and anomalies of neuronal migration: MRI and clinical aspects. Acta Neurol Scand 1992;86:24-32

13. Kuzniecky RI. MRI in cerebral developmental malformations and epilepsy. Magn Reson Imaging 1995;13:1137-45

14. Raymond AA, Fish DR, Sisodiya SM, et al. Abnormalities of gyra- tion, heterotopias, tuberous sclerosis, focal cortical dysplasia, microdysgenesis, dysembryoplastic neuroepithelial tumour and dysgenesis of the archicortex in epilepsy. Clinical, EEG and neuroimaging features in $\mathbf{1 0 0}$ adult patients. Brain 1995;118: 629-60

15. Leventer RJ, Jansen A, Pilz DT, et al. Clinical and imaging heterogeneity of polymicrogyria: a study of 328 patients. Brain 2010;133:1415-27

16. Vinters HV, Kerfoot C, Catania M, et al. Tuberous sclerosis-related gene expression in normal and dysplastic brain. Epilepsy Res 1998;32:12-23

17. Stehbens WE. Etiology of intracranial berry aneurysms. J Neurosurg 1989;70:823-31

18. Malak R, Nguyen DK, Bojanowski MW. Non ruptured MCA aneurysms presenting with insular related symptoms. Can J Neurol Sci 2010;37:219-22

19. Cereda C, Ghika J, Maeder P, et al. Strokes restricted to the insular cortex. Neurology 2002;59:1950-55

20. Bien CG, Widman G, Urbach $\mathrm{H}$, et al. The natural history of Rasmussen's encephalitis. Brain 2002;125:1751-59

21. Granata T, Gobbi G, Spreafico R, et al. Rasmussen's encephalitis: early characteristics allow diagnosis. Neurology 2003;60:422-25

22. Chiapparini L, Granata T, Farina I, et al. Diagnostic imaging in 13 cases of Rasmussen's encephalitis: can early MRI suggest the diagnosis? Neuroradiology 2003;45:171-83

23. Wagner J, Schoene-Bake JC, Bien CG, et al. Automated 3D MRI volumetry reveals regional atrophy differences in Rasmussen encephalitis. Epilepsia 2012;53:613-21

24. Taillandier L, Duffau H. Epilepsy and insular grade II gliomas: an interdisciplinary point of view from a retrospective monocentric series of $\mathbf{4 6}$ cases. Neurosurg Focus 2009;27:E8

25. Caveness WF. Epilepsy, a product of trauma in our time. Epilepsia 1976;17:207-15

26. Annegers JF, Hauser WA, Coan SP, et al. A population-based study of seizures after traumatic brain injuries. $N$ Engl J Med 1998;338:20-24

27. Pomeroy SL, Holmes SJ, Dodge PR, et al. Seizures and other neurologic sequelae of bacterial meningitis in children. $N$ Engl J Med 1990;323:1651-57

28. Gupta S, Naheedy M, Elias D, et al. Post infarction seizures: a clinical study. Stroke 1988;19:1477-81

29. Aghakhani Y, Rosati A, Dubeau F, et al. Patients with temporoparietal ictal symptoms and inferomesial EEG do not benefit from anterior temporal resection. Epilepsia 2004;45:230-36

30. Harroud A, Bouthillier A, Weil A, et al. Temporal lobe epilepsy surgery failures: a review. Epilepsy Res Treat 2012;201651 